\title{
LES CASQUES COOLUS-MANNHEIM DE VIEILLE-TOULOUSE. REMARQUES SUR LES TRANSFERTS D’ARMEMENT A L'EPOQUE REPUBLICAINE DANS LES PROVINCES OCCIDENTALES
}

\author{
POR
}

\author{
ALEXIS GORGUES
}

\section{RÉSUMÉ - RESUMEN}

On s'interroge dans cet article sur la signification des casques d'origine italique trouvés dans deux puits de Vieille-Toulouse (Haute-Garonne, France), dans des niveaux du milieu du 1er s. av. J.-C. Ces éléments de panoplie peuvent être mis en rapport avec un texte de César ( $B G$ III, 20) dans lequel des nobles tolosates sont appelés pour une campagne militaire, probablement sur fond de liens de clientèle.

Se busca en este artículo el significado de los cascos de origen itálico que fueron encontrados en dos pozos de Vieille-Toulouse (Haute-Garonne, Francia), en niveles de mediados del siglo I.a.C. Se establece una posible relación entre estos elementos de panoplia y un texto del Bellum Gallicum (III, 20) en el que nobles de Tolosa son llamados por César para una campaña militar, en un marco de lazos personales de tipo clientelar.

\section{MOTS CLÉS - PALABRAS CLAVE}

Armement, casque Coolus-Mannheim, Vieille-Tolouse, Guerre des Gaules, clientèle.

Armamento, casco Coolus-Mannheim, Vieille-Toulouse, Guerra de las Galias, clientela.

Le site de Vieille-Toulouse (Haute-Garonne, France), vaste habitat localisé sur les coteaux surplombant la Garonne non loin de sa confluence avec l'Ariège (fig. 1), a livré au cours des fouilles menées par Michel Vidal dans les années 1960-1970 (bilan dans Vidal, 2001, 102-128) un certain nombre d'éléments liés au domaine guerrier ${ }^{1}$, principalement datables du I ${ }^{\text {er }}$ s. av. J-C. A cette époque, ce site, occupé depuis le début du II $^{\mathrm{e}}$ s. av. J-C., est le plus important du Toulousain; l'agglomération de plaine de Saint-Roch (Toulouse) semble en effet être abandonnée aux alentours de -75 .

Nous nous concentrerons ici sur une partie spécifique de ce mobilier, constituée par des casques d'origine italique découverts dans la partie basse du comblement d'un certain nombre de puits. Ces puits, chargés d'une historiographie particulièrement dense, ont été successivement qualifiés de «funéraires», de «rituels», ou encore (et c'est de loin le terme le moins

1 Ce mobilier fait actuellement l'objet d'une étude de M. Schönfelder (RGZM, Mayence) avec la collaboration de l'auteur de ces lignes, dans le cadre de l'ACR intitulée «Aux origines de Tolosa», dirigée par M. Vidal. L'article présenté ici reprend et approfondit la contribution de l'auteur à un article réalisé en collaboration avec M. Schönfelder, intitulé «Militaria d'époque césarienne à Boé (Lot-et-Garonne) et à Toulouse (Haute-Garonne) - quelques considérations», à paraître dans la série Bibracte. 
adapté) de «puits à amphores». Récemment, P. Moret et l'auteur de ces lignes ont proposé une interprétation fonctionnelle de ces structures, en déduisant de l'étude de leur comblement qu'il devait s'agir de puits à eau, secondairement réutilisés comme dépotoirs (Gorgues, Moret, 2003).

La présence de ces pièces d'armement défensif italiques dans un contexte indigène suscite un certain nombre d'interrogations concernant les transferts d'armement à une époque où, rappelons-le, Tolosa appartient à la province de Gallia Transalpina depuis -121 (si on suit la chronologie classique) ou successivement aux provinces d'Hispania Citerior (de -197 aux alentours de -75 , si on suit l'hypothèse d'Ebel 1976) puis de Gallia Transalpina, et se trouve donc sous contrôle romain. Plus largement, ces découvertes soulèvent le problème des modalités d'adoption de pièces d'armement italiques dans le cadre de panoplies indigènes dans les provinces occidentales (Hispania et Gallia Transalpina) au cours de la période républicaine. Curieusement, cette question, en ce qui concerne Toulouse et plus largement le Midi de la Gaule, n'a jamais fait l'objet de discussions, du fait que le contexte général n'a jamais été pris en considération.

Cet article ne constitue donc pas une tentative de révision du mobilier d'un site spécifique, mais une contribution à l'étude des interactions entre monde indigène et monde romain à la fin de l'époque républicaine.

\section{LES CASQUES: CONTEXTES DE DÉCOUVERTE ET DATATIONS}

Les casques d'époque républicaine découverts à Vieille-Toulouse sont au nombre de cinq. L'attribution typochronologique d'un autre casque découvert dans le puits 9 du site voisin d'Estarac (Vidal 1991,188) pose un certain nombre de problèmes. Nous le laisserons ici provisoirement de côté, de même que les particularités typologiques des casques et les modifications dont ils ont fait l'objet, aspects qui sont actuellement en cours d'étude (voir note 1$)$.

Trois casques en bronze, appartenant à la variante légère des casques de type CoolusMannheim, furent découverts dans la partie basse du puits 23 de Vieille-Toulouse (fig. 2; sur ce puits, Vidal 1991, 178-179), associés à de nombreuses pièces de vaisselle métallique, dont six situles, et des petits objets, parmi lesquels un harnachement. Cette partie du comblement correspond sans doute à la couche de fonctionnement du puits, constituée d'une accumulation de restes végétaux sur $2 \mathrm{~m}$ de haut. Cette couche ne peut pas être datée avec précision du fait de l'absence de mobilier dont la chronologie puisse être établie avec précision, mais il est possible de dater les couches supérieures du comblement, qui ont livré un mobilier abondant. Le faciès d'importation est dominé par les productions de campaniennes $\mathrm{B}$ de Calès (aussi appelées «B-oïdes»). En l'absence des productions les plus tardives de l'époque républicaine (principalement les productions de céramiques à vernis noir à pâte grise dérivées de la campanienne B) et de productions d'époque impériale (notamment les sigillées italiques), il est possible de dater ce comblement des années $-75 /-50$.

Le puits 16 a livré pour sa part deux casques Coolus-Mannheim (fig. 3): un de la variante légère et un de la variante lourde, dans une couche inférieure qui contenait également une pointe de lance, une serpe et des pièces de vaisselle en bronze. Ces objets étaient répartis en plusieurs groupes au sein d'un comblement «de blocs de marne sableuse provenant de l'effondrement de trois des parois», surplombé d'une couche de végétaux qui a livré, entre autres éléments, un demi-mors de cheval (Vidal 1991, 172-176; nous interprétons ces couches de végétaux comme la partie basse des rejets d'ordures qui servirent à combler le puits). Si pour des raisons identiques à celles énoncées pour le puits 23 , la chronologie de la couche inférieure ne peut être fixée avec précision, celle de son comblement peut faire l'objet d'une 
datation assez précise. Celle-ci à d'ailleurs suscité une polémique basée sur l'appartenance ou non à ces couches d'un bol en sigillée italique de Vibius Scrofula daté des années -15/-10 (voir Vidal 2001, 129, note 79; Benquet et al. 2001, 134; première discussion sur la provenance de ce vase dans Vidal, 1991, 174). Dans l'opinion de M. Vidal, ce vase trouvé entier pourrait être une intrusion et le puits serait selon lui d'époque républicaine. Or, il faut signaler que même si ce vase est intrusif, d'autres éléments (dont une autre estampille de Scrofula, conservée dans les réserves du Musée de St-Raymond, mais aussi une estampille anépigraphe des ateliers de Bram et une imitation en céramique grise indigène de bol en sigillée italique) montrent clairement que ce comblement s'est constitué à l'époque augustéenne, même si l'essentiel du mobilier est plus ancien et renvoie aux années -75/-50 (Benquet et al. 2001, 134; Gorgues, Moret 2003, 135; Gorgues, à paraître).

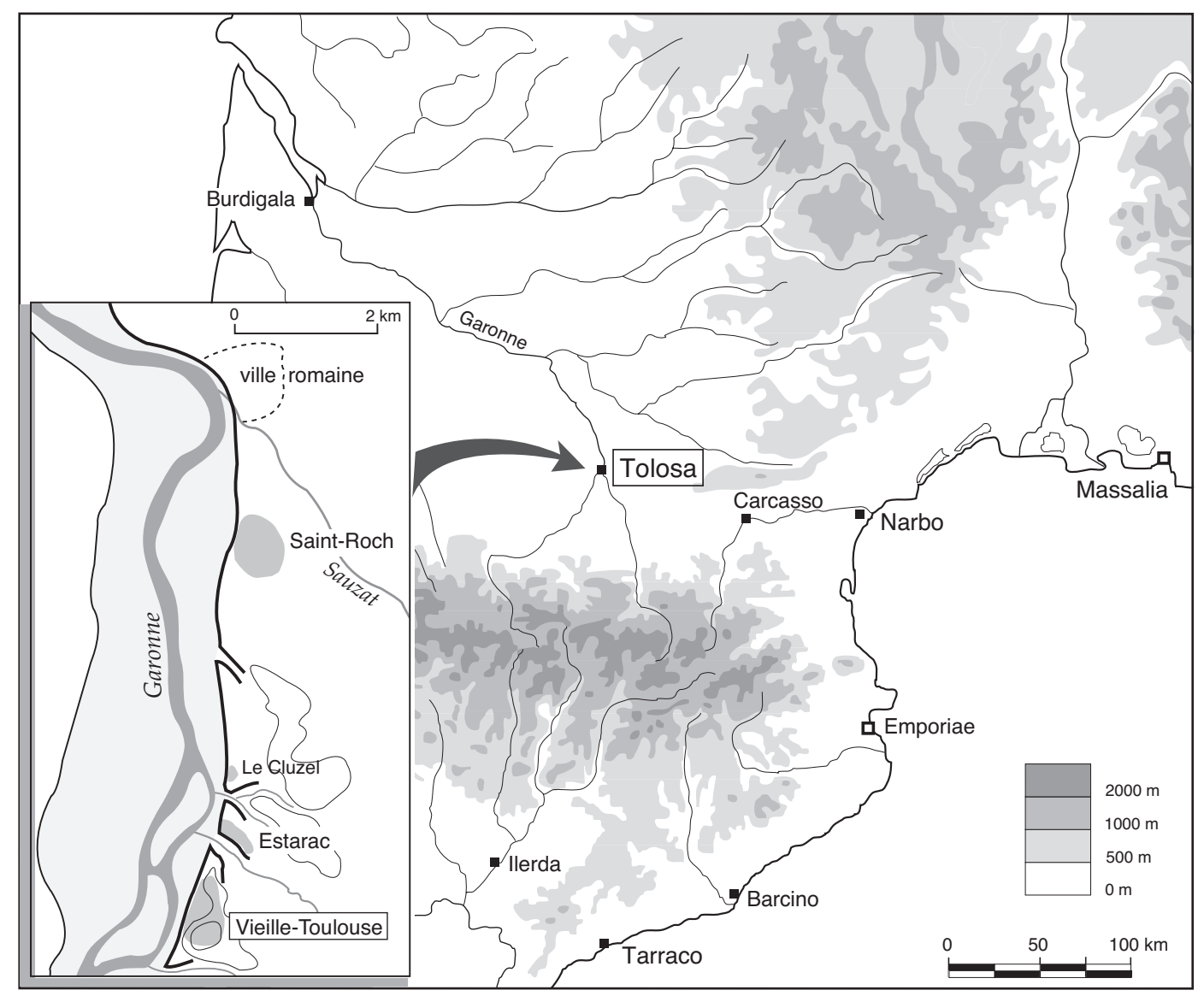

Fig. 1. Localisation de Vieille-Toulouse et des principaux sites de la fin de l'Age du Fer du sud de l'agglomération toulousaine (fig. P. Moret).

Première observation: le phénomène ayant entraîné la présence d'armes dans les puits du Toulousain est marginal (il n'affecte que deux puits, sur environ cinquante puits et fosses fouillés sur le site) et il est relativement tardif (c'est sans doute ce qui explique le fait qu'on n'en retrouve pas à Saint-Roch, site qui est abandonné à cette époque). Un nombre réduit de puits a livré un mobilier privilégié. 
Ce fait peut soutenir deux interprétations différentes mais pas forcément contradictoires. La première serait que l'on se trouve face à des dépôts organisés liés au processus de construction et de mise en service du puits (hypothèse ébauchée dans Gorgues, Moret 2003, 138); dans le cas qui nous intéresse, le puits aurait été construit à l'initiative de personnes possédant armes et mobilier de bronze, qu'ils auraient déposés dans le cadre d'un sacrifice propitiatoire. La seconde interprétation, qui a, à titre provisoire, notre préférence, est qu'il s'agissait d'objets liés à l'activité de puisage: casque récupérés et transformés en seaux, situles, etc., tombés au fond du puits à cause d'une rupture de la corde les reliant à la surface. Dans ce cas, la présence de mobilier privilégié dans un petit nombre de puits contrastant avec une foule d'autres puits au comblement plus banal tendrait à démontrer l'usage préférentiel d'un puits par un groupe spécifique de personnes, de sorte que le comblement des puits serait le reflet -certes déformé par le contexte- de la «culture matérielle» du groupe. Dans le cas des puits 16 et 23, ce groupe détiendrait des armes et de la vaisselle en bronze en nombre suffisant pour en utiliser une partie (une fois hors d'usage dans le cas des armes) dans le cadre d'une activité de puisage. Cette interprétation peut elle-même conduire à deux conclusions possibles. Ce groupe spécifique pourrait être identifié comme une famille privilégiée: le puits serait alors à interpréter comme une structure privée, et son comblement comme le reflet du mobilier utilisé pour le puisage par l'unité domestique possédant le terrain sur lequel il se trouve. Seconde hypothèse: l'eau puisée dans ces puits aurait servi à un usage spécifique, rituel, ce qui expliquerait qu'on la puisait avec un mobilier spécifique. Dans ce cas, le mobilier de puisage devrait être interprété comme lié à un groupe social (celui qui pratiquait ce rituel lié à l'eau).

Evidemment, ces hypothèses restent très ouvertes et permettent d'envisager un grand nombre de variantes ou de nuances: puits à eau ayant fait l'objet d'un dépôt inaugural pour favoriser la réussite d'une entreprise «domestique»; ou bien dépôt lié à un usage spécifique des eaux.... Quoi qu'il en soit, l'aspect localisé de ces découvertes renvoie sans ambiguiité à une association entre ces deux puits et un groupe spécifique (famille ou groupe social), détenteur d'armes italiques. Dès lors, on peut se demander quel était ce groupe, et comment il est entré en possession de ces armes.

\section{L'IDENTITÉ DES PORTEURS DE CASQUE}

Se pose en premier lieu la question du statut de ces individus: s'agissait-il de citoyens romains, autrement dit de légionnaires, ou d'indigènes? Qu'il se soit agi de citoyens romains paraît peu vraisemblable pour deux raisons. Tout d'abord, le contexte général est celui d'une grande agglomération indigène, et le contexte spécifique celui de puits dont le comblement renvoie à une culture matérielle locale. Ensuite, le légionnaire post-marien maintient avec sa panoplie une relation qui rend difficilement imaginable le dépôt d'armes ou leur réutilisation sous forme de seau à puiser. C'est un armement qui lui est fourni par l'Etat, et qu'il paye sur sa solde (Goldsworthy 2005, 47), contrairement aux légionnaires «miliciens» qui en étaient propriétaires. De fait, la présence de casques du $\mathrm{I}^{\mathrm{er}} \mathrm{s}$. av. J-C. dans des contextes romains où leur perte ne peut être liée à un facteur accidentel est extrêmement rare ${ }^{2}$. En revanche, dans le monde indigène de la Méditerranée Occidentale, l'association entre guerrier et panoplie est telle qu'elle se maintient, au moins partiellement, jusque dans l'au-delà. Après Marius (mais peut être aussi avant), la limite entre domaine guerrier et domaine civil, en terme de culture matérielle, est nettement plus perméable dans les sociétés indigènes que dans la so-

2 Sur la présence d'armes au sein de sanctuaires en Italie, on se rapportera par exemple à la récente synthèse de Gabaldón Martínez, 2004, 171-265. 
ciété romaine. La réutilisation ou le dépôt d'armes dans les profondeurs d'un puits par un individu donné semble une pratique plus cohérente avec ce que l'on sait du monde indigène qu'avec le monde romain ${ }^{3}$.

Une relation directe entre le propriétaire du casque et le contexte de découverte peut donc être supposée avec un degré élevé de vraisemblance: ces armes équipaient des guerriers indigènes. Le service auxiliaire offre un cadre dans lequel des soldats gaulois vont combattre au sein de formations militaires romaines, ce qui fournit déjà un schéma d'explication général au problème de la présence d'armes romaines en contexte indigène. Cependant, l'association déjà évoquée de ces armes au sein d'ensembles liés à l'activité d'un groupe spécifique pose le problème de l'identité de ces auxiliaires (toute personne en état de combattre et membre d'une communauté alliée de Rome pouvait servir à ce titre, ce qui laisse une marge d'incertitude importante) et de leur place au sein de la société indigène.

Un texte chronologiquement proche des éléments commentés ici permet d'éclairer ce problème. César relate ainsi les préparatifs de l'invasion de l'Aquitaine par Crassus, en 56 av. n-è. (Guerre des Gaules, III, 20):

«Il (Crassus) fit donc ses provisions de blé, rassembla des auxiliaires et de la cavalerie, convoqua en outre individuellement, de Toulouse, Carcassonne et Narbonne, cités de la province qui sont voisines de l'Aquitaine, un grand nombre de soldats éprouvés; puis il pénétra sur le territoire des Sotiates» (trad. Constans, Les Belles Lettres).

«Itaque re frumentaria prouisa, auxiliis equitatuque conparato, multis praeterea uiris fortibus Tolosa, Carcasone et Narbone, quae sunt ciuitates Galliae prouinciae finitimae [ex] his regionibus, nominatim euocatis in Sotiatum fines exercitum introduxit».

La première partie du texte, qui évoque les préparatifs de la campagne, indique des rapports aux populations locales qui ne diffèrent que peu de ce que l'on peut observer en Gaule chevelue: on rassemble des vivres et on demande aux alliés une contribution en hommes, auxiliaires et cavaliers ( $c f$. par exemple Guerre des Gaules, I, 15-16 pour un ravitaillement en blé chez les Eduens, qui fournissent de plus de la cavalerie). Cependant, l'originalité de ce passage est constituée par la dernière mesure que prend Crassus, la convocation individuelle de guerriers d'élite, qui n'apparaît pas lorsque César évoque les rapports entre l'armée romaine et les alliés de Gaule continentale. L'expression vir fortis est utilisée une autre fois telle quelle dans la Guerre des Gaules $(\mathrm{V}, 35)$ et deux fois avec fortis au superlatif (II, 25 et $\mathrm{V}, 44)$. Dans ces trois cas, la formule s'inscrit dans une description de bataille, et vise à mettre en valeur des individus cités pour leur exceptionnel courage dans l'action. Ce fait pourrait laisser croire que derrière cette formule se cache un rappel de légionnaires vétérans résidant entre Narbonne et Toulouse. La probabilité existe (même si elle semble plus élevée concernant Narbonne, colonie romaine, que Toulouse ou Carcassonne, des villes indigènes), mais doit être pondérée.

En effet, le contexte du chapitre III, 20 est très éloigné de celui des trois autres mentions. En revanche, un parallèle proche peut être trouvé dans la Guerre Civile (I, 39): alors qu'il relate des évènements advenus au cours de la campagne d'Espagne, César applique à des nobles de Gaule chevelue une formule proche de celle qu'il avait employée dans la Guerre des Gaules pour les evocati de Transalpine:

«Les troupes auxiliaires comprenaient aux alentours de six mille fantassins et trois mille cavaliers, qui avaient servi sous son commandement dans toutes les guerres précédentes, et il en avait fait venir autant

3 De fait, les parallèles à l'échelle régionale ne manquent pas, à Agen (Boudet 1996, 9-20 et 54-73) et VicFezensac par exemple. 
de la Gaule, qu'il avait pacifiée, où il avait appelé individuellement tous les hommes les plus nobles et valeureux de chaque cité» (trad. P. Fabre, Les Belles Lettres).

«Auxilia peditum ad VI milia, equitum III milia, quae omnibus superioribus bellis habuerat, et parem ex Gallia numerum, quam ipse pacauerat, nominatim ex omnibus civitatibus nobilissimo et fortissimo quoque euocato».

Ce passage est très clairement parallèle de celui du livre III de la Guerre des Gaules: les troupes fournies par les Gaulois comportent des auxiliaires d'infanterie et de cavalerie. Une catégorie spécifique se détache cependant, celle des hommes appelés individuellement, «très nobles et très valeureux», une formule très proche de celle utilisée à propos des préparatifs de Crassus. Ce passage démontre que l'evocatio individuelle peut s'adresser à des Gaulois, des nobles selon toute vraisemblance. On peut donc voir dans le passage de la Guerre des Gaules l'attestation non pas d'un rappel de légionnaire vétérans mais celle d'une demande envoyée (par lettre ou par messager) à des nobles indigènes résidant sur les territoires des cités romaines de l'ouest de la Transalpine, sollicitant leur concours pour une campagne spécifique, celle d'Aquitaine. Autrement dit, il s'agit un recrutement nominatif (contrairement au recrutement anonyme prévalant dans le cas des soldats prêtant un service militaire, que ce soit comme légionnaires ou comme auxiliaires), qui se basait selon toute vraisemblance sur des liens d'amicitia unissant l'officier romain et le noble indigène.

Les caractéristiques des contextes dans lesquels furent découverts les casques de type Coolus-Mannheim de Vieille-Toulouse amènent à interpréter ceux-ci comme la trace matérielle de ces liens d'amitié personnels. Leur port par des soldats indigènes, servant probablement comme cavaliers ${ }^{4}$, revêt sans nul doute un caractère pratique: le casque est un élément fort d'identification du soldat, et donner des casques spécifiquement romains à des soldats dont la panoplie est essentiellement gauloise permet de les différencier des Gaulois «ennemis» $\rangle^{5}$. Cependant, la valeur de ces casques devait être principalement symbolique. Le don d'une arme facilement identifiable (il s'agit de casques en bronze, différents des casques gaulois en fer connus par ailleurs dans la région: Schaaf 1988, 303-304; Schönfelder 2000), dans une société où le statut militaire devait jouer un rôle important, constitue une façon de «matérialiser» le lien personnel. Le casque, don d'un magistrat romain à un noble indigène qui tirait une bonne partie de son pouvoir et de son influence de sa fonction guerrière, permet non seulement de consacrer le lien qui les unit comme compagnons d'armes (c'est le sens de la convocation individuelle), mais d'afficher ce lien aux yeux de tous. Il est la reconnaissance de la place du noble dans l'ordre romain, et la démonstration de l'importance de son porteur comme relais entre sa communauté et Rome. C'est aussi le symbole de la protection qu'offre le magistrat au noble indigène en échange de ses services guerriers, devenant ainsi la marque du statut de client de ce dernier.

Bien évidemment, il ne s'agit pas ici d'affirmer que les casques de Vieille-Toulouse sont les armes offertes par César ou Crassus aux nobles Tolosates convoqués individuellement en -56 , mais plutôt de recouper les sources afin d'analyser un processus amenant à l'adoption d'armes italiques par des indigènes des provinces occidentales. En effet, le cas de Tolosa est loin d'être isolé et s'inscrit dans le contexte historique des deux derniers siècles de la République.

4 On rappellera à ce propos la présence d'un «demi-mors» (puits 16) et d'un élément de harnachement (puits 23) dans des couches que nous interprétons comme dépotoirs, ainsi que l'interprétation que nous donnons de ces rejets comme spécifiques à un groupe social ou familial réduit.

5 Ainsi, l'incident survenu au cours de la bataille de Gergovie ( $B G$, VII, 50), quand l'apparition d'auxiliaires éduens sème le trouble dans l'armée romaine, montre bien trois choses: que les Gaulois servant dans l'armée de César avaient un élément d'identification (l'épaule droite découverte); que cet élément ne suffisait pas à rassurer les légionnaires; que la norme était que les auxiliaires soient dotés d'une panoplie indigène et que le port d'éléments d'armement romains semble marginal. 


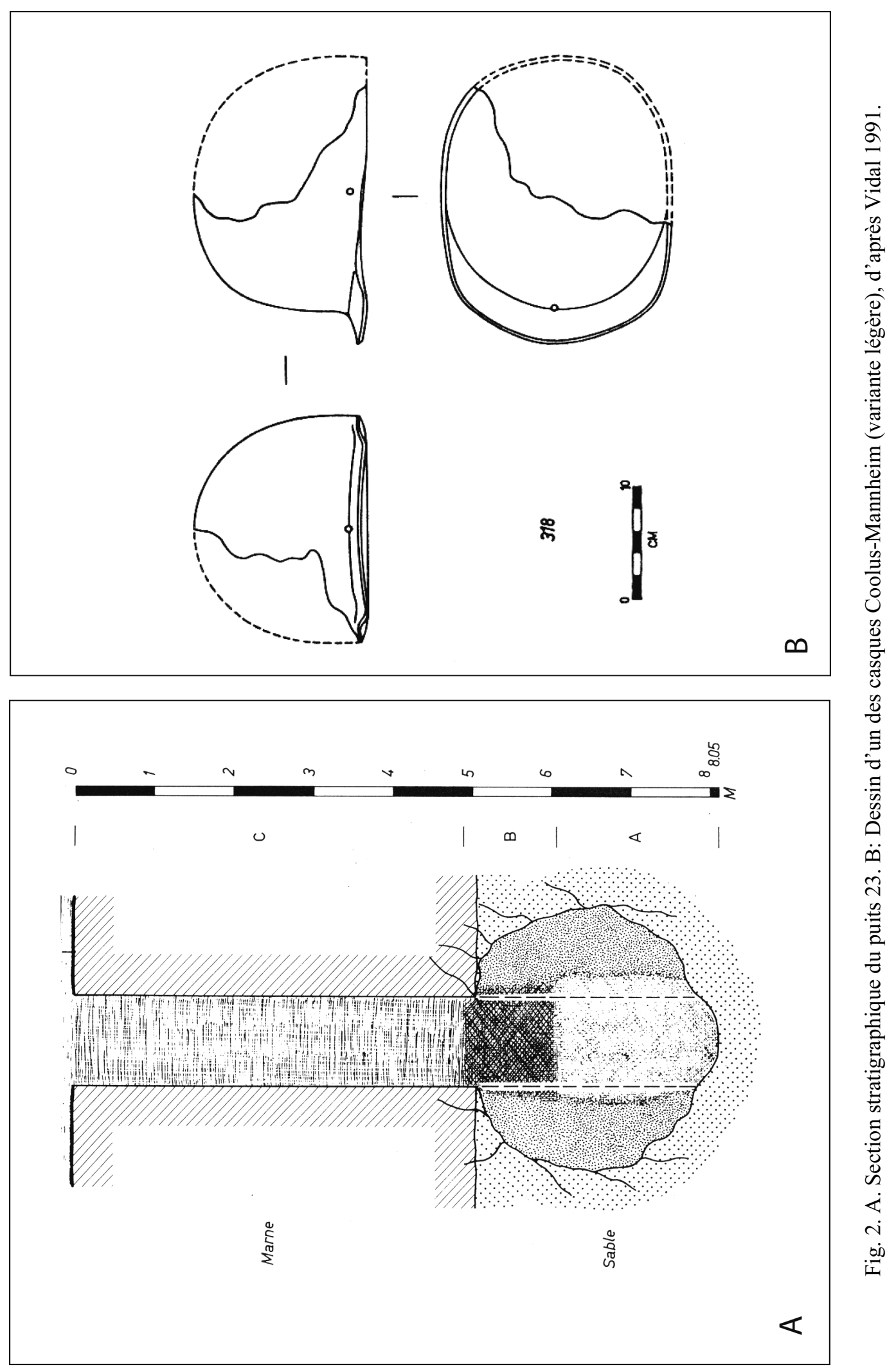


FOREIGN CLIENTELAE ET TRANSFERTS D’ARMEMENT DANS LES PROVINCES OCCIDENTALES: UNE HYPOTHĖSE EXPLICATIVE PARTIELLE

L'adoption d'armes italiques par des sociétés de tradition guerrière différente est un phénomène qui commence, en Occident, par la diffusion de casques de type Montefortino à la fin du III ${ }^{\mathrm{e}}$ s. av. J.-C. ${ }^{6}$. Il s'agit du principal type de casque métallique présent dans les contextes ibériques des années de la fin du $\mathrm{III}^{\mathrm{e}} \mathrm{s}$. et du $\mathrm{II}^{\mathrm{e}} \mathrm{s}$. av. J-C., alors que cet équipement avait quasiment disparu de la panoplie indigène ${ }^{7}$. Ce fait souligne à lui seul l'influence du contexte historique dans l'adoption de pièces d'armement exogènes par les sociétés de la fin de l'Age du Fer.

En ce qui concerne les découvertes en contexte ibérique, et principalement celles en milieu funéraire qui se concentrent dans les régions du Levante, à l'exception notable des découvertes de la nécropole ampuritaine de Les Corts, F. Quesada $(1997,560)$ a raison de souligner que c'est le caractère spécifique de chaque cas qui domine, au détriment d'une explication générale. La diversité des contextes de découverte montre que le casque n'est pas l'attribut d'un statut social (celui d'un chef, par exemple), mais bien un attribut personnel, un élément de panoplie lié à l'histoire du guerrier.

Cette constatation, si elle laisse la place à d'autres possibilités, n'est en rien contradictoire avec notre hypothèse: le lien de clientèle est avant tout un lien d'individu à individu, dont l'établissement est lié, dans le cas qui nous occupe, au rôle, à l'influence et au statut du client dans sa communauté d'origine, impossible à évaluer d'après des ensembles funéraires dont les modalités de constitution nous échappent et ne renvoient pas forcément au statut réel que le défunt avait de son vivant.

L'importance et les caractères de la constitution d'une clientèle locale dans les premières années de l'occupation romaine de l'Hispania, principalement par Scipion l'Africain, ont déjà été soulignés par Badian (1958, 119), qui a bien analysé le point de vue indigène d'un tel processus: ces liens unissent le noble indigène à un individu, non à un magistrat ou à une institution. Le don (et le contre-don), dans un rapport perçu par les indigènes comme une relation entre nobles, peut dans ce contexte revêtir une importance particulière. Or, il apparaît clairement, notamment à la lumière des récits des Guerres Civiles, que cette attitude ne changera pas jusqu'à la fin de la République. Ainsi, le passage cité plus haut du Bellum Civile $(\mathrm{I}, 39)$ montre que les clientèles gauloises et hispaniques de César vont le suivre dans la guerre contre Pompée sans se poser le problème de savoir qui des deux détient la légitimité institutionnelle. La constitution de clientèles étrangères, sous couvert d'exercer les fonctions inhérentes au gouvernement des provinces, va ainsi devenir un des éléments des jeux du pouvoir entre la période des Gracques et celle d'Auguste (Badian 1958, 252-284).

Un tel phénomène est extrêmement multiforme. La citoyenneté devait être, au moins au $\mathrm{I}^{\mathrm{er}}$ s. av. J-C., un enjeu de taille, pour celui qui la reçoit comme pour celui qui la confère. L'édit d'Ascoli pris en -90 ou -89 par Cn. Pompeius Strabo afin de conférer la citoyenneté romaine aux cavaliers de la turma Salluitana, du fait de leur valeur au combat lors du siège d'Asculum (Criniti, 1970), montre à plusieurs niveaux l'importance de cet aspect. Tout d'abord, rappelons qu'il s'agit de cavaliers, sans doute de «nobles», en tout cas issus d'une catégorie sociale capable de mobiliser les richesses nécessaires à l'achat, l'entraînement et l'équipement de chevaux de guerre, et que c'est par le service militaire qu'ils acquièrent leur nouveau statut de citoyens romains. F. Quesada $(1998,178)$ a d'ailleurs formulé l'hypothèse qu'il pouvait s'agir, là aussi, de soldats evocati. Tous ces individus recevront le prénom et le

6 On prend ici en compte la révision chronologique du casque Montefortino de la sépulture 4F/2 de Pozo Moro: F. Quesada (1997, 562), a bien montré que ce casque, dont le couvre-nuque porte l'inscription latine Mulus (De Hoz 1994), doit être daté de la fin du $\mathrm{III}^{\mathrm{e}} \mathrm{s}$. av. J-C ou plus probablement du $\mathrm{II}^{\mathrm{e}} \mathrm{s}$.

7 Sur ces casques et leur présence en péninsule Ibérique, on suit ici le travail de Quesada 1997, 554-564. 

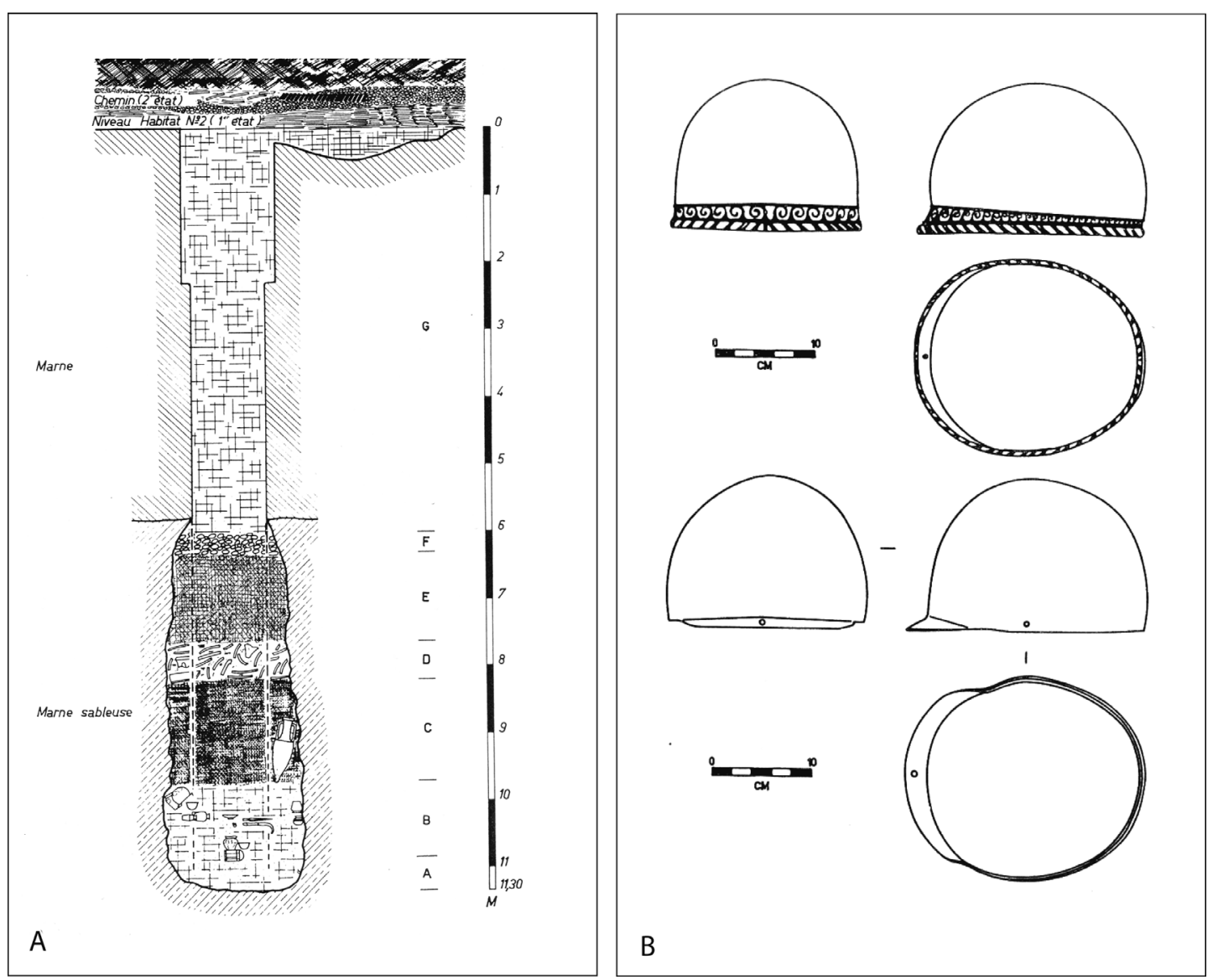

Fig. 3. A. Section stratigraphique du puits 16. B: en haut, dessin du casque Coolus-Mannheim variante lourde; en bas, dessin du casque de la variante légère, d'après Vidal 1991.

gentilice de Cn. Pompeius, et rentreront, comme s'il s'agissait d'affranchis, dans sa clientèle personnelle. Enfin, il est particulièrement intéressant de constater que les cavaliers ilergètes de cette turma reçoivent la citoyenneté romaine alors qu'ils portent déjà des formules onomastiques latines (Q. Otacilius Suisetarten f., Cn. Cornelius Nesille f., P. Fabius Enasagin f.). Pour expliquer ce fait, on ne peut qu'émettre l'hypothèse que ces individus portaient (et usurpaient) un nom romain pour renforcer leur influence au sein de leur communauté. En Gaule, des citoyens romains d'origine indigène figurent dans le conseil de César, comme l'Helvien C. Valerius Caburrus (B.G., IV, 12), preuve de l'importance de l'élément militaire dans ce processus.

Les relations personnelles non sanctionnées ou indirectement sanctionnées par une décision juridique devaient également être importantes. Avoir l'oreille et la protection d'un Romain influent, promagistrat d'abord, sénateur ensuite, était une garantie d'influence au niveau local pour les potentats indigènes, qu'ils résident dans les provinces ou dans des zones périphériques. Ainsi, être parrainé par un influent citoyen romain permettait à des chefs indigènes de recevoir du Sénat le titre d' «ami du peuple romain»» ${ }^{8}$. La contrepartie de ces fa-

8 Par exemple, dans la Guerre des Gaules: Catamandoelis (I, 3), Arioviste (I, 35) ou encore le père de Pison l'Aquitain (IV, 12). 
veurs était surtout, sans doute, la prestation du service militaire, dans un contexte où les armées servaient de plus en plus un personnage avant de servir un Etat.

La souplesse de ces pratiques oblige à faire preuve de prudence au moment d'interpréter les données archéologiques: ainsi, il est exclu de prétendre que tel ou tel casque ait appartenu à un indigène fait citoyen romain, mais il est licite de les interpréter, dans certains contextes, comme la trace matérielle de relations privilégiées entre élites indigènes et grandes familles romaines. On est parti de Vieille-Toulouse pour développer ce thème, car dans ce cas les sources écrites éclairent partiellement le contexte archéologique. On aurait aussi pu partir d'Agen, où des découvertes comparables renvoient probablement au même phénomène ${ }^{9}$, à la différence près que l'on se trouve là hors du territoire provincial.

Ceci ne veut pas dire que toute découverte d'armement romain en contexte indigène peut être interprétée dans le même sens, ni, surtout, que l'on peut donner à ces découvertes une signification événementielle. Pour citer un seul obstacle, l'impossibilité de fixer des chronologies précises à moins de vingt-cinq ans (le temps séparant par exemple les guerres sertoriennes des guerres civiles) et la difficulté de caractériser certaines périodes (la période -50/40, par exemple) doit nous obliger à la prudence. La portée de l'interprétation proposée ici est avant tout structurelle: ces casques (mais d'autres éléments aussi, sans doute) sont, dans des contextes donnés, la manifestation en termes de culture matérielle des liens se développant entre élites indigènes et dynastes romains.

Curieusement, l'impact des relations sociales dans les modifications de la culture matérielle est quasiment ignoré par le discours archéologique pour les périodes envisagées ici, alors que c'est un aspect dominant (parfois de façon excessive) lorsqu'on s'intéresse aux périodes plus anciennes. Le don et le contre-don, les relations centre-périphérie, etc., sont par exemple des éléments clés du discours archéologique récent portant sur les régions celtiques à l'époque halstattienne et au début de La Tène. Pour la fin de l'Age du Fer, les explications qui ont actuellement cours ont un fond conjoncturel (trophées, butin...) ou commercial (ce dernier point dominant de façon générale le discours sur les bouleversements de la culture matérielle à la fin de l'époque républicaine). Cet état de fait est particulièrement surprenant, alors même que l'on se trouve à une époque où les relations sociales deviennent particulièrement intenses et où elles sont très structurées, à la fois d'un point de vue pratique et juridique. Le rôle de ces relations, dans certains domaines - et le domaine militaire semble particulièrement sensible à de tels phénomènes- dut être très significatif, et devrait être revalorisé.

Alexis Gorgues

Casa de Velázquez

\section{ABRÉVIATIONS BIBLIOGRAPHIQUES}

Badian, E. (1958): Foreign Clientelae (264-70 B.C.), Oxford, Clarendon Press, 1958.

Benquet L.; Boube, Chr.; Domergue, C.; Hesnard, A.; Moret, P.; Passelac, M.; Scheers, M. (2001): «Cinq puits de Vieille-Toulouse: mobilier et chronologie», in Pailler, 2001, pp. 129-155.

BOUdET, R. (1996): Rituels celtes d'Aquitaine, Paris, 1996.

9 Le puit 41 du site de l'Ermitage à Agen a livré deux casques de la variante légère du type Coolus-Mannheim et le puits Z1 en a livré un (Boudet 1996, 22-31 et 54-73; Verdin dans Gaulois 2004, 36-41, Verdin, Vidal dans Gaulois 2004, 52-55). 
Criniti, (N.) 1970: L'epigrafe di Asculum di Gn. Pompeo Strabone, Publicazione dell'università cattolica del S. Cuore, Milan, 1970.

De Hoz, J. (1994): «Una probable inscripción latina en un casco de Pozo Moro», Archivo Español de Arqueología, 67, pp. 223-227.

EBEL, C. (1976): Transalpine Gaul. The emergence of a Roman Province, Brill, Leiden, 1976.

FEugère, M. (1994): «L'équipement militaire d'époque républicaine en Gaule», Military equipment in context, Journal of Roman Military Equipment Studies, 5, 1994, pp. 3-23.

Gabaldón Martínez, M. del M. (2004): Ritos de armas en la Edad del Hierro. Armamento y lugares de culto en el antiguo Mediterráneo y el mundo celta, Anejos de Gladius, 7, Madrid, 2004.

GAULOIS (2004): Gaulois des pays de Garonne. IIe-Ier s. avant J-C., Guide de l'exposition, Toulouse, Musée Saint-Raymond, 2004.

Gorgues, A.; MoRet, P. (2003):«Toulouse et Vieille-Toulouse», in Cultes et sanctuaires en France à l'Age du Fer, Gallia, 60, 2003, pp. 107-138.

Gorgues, A. (2004): «Les pratiques culinaires à Vieille-Toulouse au Ier s. av. J-C. et leur contexte régional», à paraître dans les actes du XXVIII ${ }^{\mathrm{e}}$ colloque de l'Association Française pour l'Etude de l'Age du Fer, Toulouse, mai 2004.

Goldsworthy, A. (2005): El ejército romano, Madrid, Akal, 2005.

PAILlER, J-M. (dir.) (2001): Tolosa. Nouvelles recherches sur Toulouse et son territoire dans l'Antiquité, Collection de l'Ecole Française de Rome, 281, Rome, 2001.

QUESADA SANZ, F. (1997): El armamento ibérico. Estudio tipológico, geografico, funcional, social y simbólico de las armas en la Cultura Ibérica (siglos VI-I a.C.), Monographies Instrumentum, 3, Montagnac, 1997, 2 vol.

QUESADA, F. (1998): «Aristócratas a caballo y la existencia de una verdadera «caballería» en la cultura ibérica: dos ámbitos conceptuales diferentes», Los Iberos, principes de Occidente. Las estructuras de poder en la sociedad ibérica, Saguntum-PLAV, extra-1, 1998, pp. 169-184.

SchaAfF, U. (1988): «Keltische Helme», Antike Helme. Sammlung Lipperheide und andere Bestände des Antikenmusums Berlin, RGZM Monographien 14, Mainz 1988, pp. 293-317.

SCHÖNFELDER, M. (2000): «Le mobilier métallique de la tombe à char tardo-celtique de Boé (Lotet-Garonne)», Aquitania 17, 2000 (2002), pp. 59-81.

VIDAL, M. (1991): «La vaisselle tardo-républicaine en Gaule du sud-ouest. Chronologie et fonction, d'après les contextes clos», in La vaisselle tardo-républicaine en Bronze (actes de la table-ronde du CNRS, Lattes, 1990), Dijon, 1991, pp. 169-191.

VIDAL, M. (2001): VIDAL «Vieille-Toulouse. Le site et ses vestiges», in Pailler, dir., 2001, pp. 102-128. 